\title{
Lorentz invariance on trial in the weak decay of polarized atoms
}

\author{
Stefan E. Müller
}

Published online: 31 January 2012

(C) The Author(s) 2012. This article is published with open access at Springerlink.com

\begin{abstract}
The invariance of the laws of physics under Lorentz transformations is one of the most fundamental principles underlying our current understanding of nature. In theories trying to unify the Standard Model with quantum gravity, this invariance may be broken, and dedicated high-precision experiments at low energy could be used to reveal such suppressed signals from the Planck scale. We will test Lorentz invariance searching for a dependence of the decay rate of spin-polarized nuclei on the daily, yearly or deliberate re-orientation of the spin. Observation of such a dependence would imply a breakdown of Lorentz invariance.
\end{abstract}

Keywords Lorentz invariance $\cdot$ Optical pumping $\cdot \beta^{+}$-decay

\section{Introduction}

The invariance of physical laws under rotations in 3-dimensional space and velocity changes (boosts) of the reference frame of a physical system is commonly referred to as Lorentz invariance. Lorentz invariance is a basic building block of both the theory of Special Relativity, as well as the local quantum field theories that build the Standard Model of particle physics. In addition, Lorentz invariance is a local symmetry of General relativity, and is connected to CPT invariance via the CPT theorem which states that a Lorentz invariant local quantum field theory must also be invariant under CPT-transformations. It has been proven that any theory which violates CPT should also violate Lorentz invariance [1] (however, this argument does not necessarily hold the other way around). Certain theoretical models of quantum gravity contain terms which violate Lorentz invariance, and this could lead to manifestations of Lorentz invariance violation also in low-energy observables accessible in laboratory experiments. Kostelecký and coworkers have developed a

S. E. Müller $(\varangle)$

Kernfysisch Versneller Instituut, Zernikelaan 25, 9747AA Groningen, The Netherlands e-mail: S.Mueller@kvi.nl 
theoretical framework named "Standard Model Extension" (SME) that contains all the properties of the Standard Model and General Relativity, but additionally contains all terms violating Lorentz invariance and CPT symmetry via hidden background fields connected to the vacuum [2]. There are many experimental tests constraining the parameters of the SME [3], most of which are tests of QED or gravity and astrophysical observations. The weak sector is tested mostly in CPT tests of neutral meson or neutrino oscillations. The electroweak decay sector is up to now largely unexplored.

In a simplified phenomological description, the differential decay rate of a weakly decaying nucleus with nuclear spin $\mathbf{I}$ can be written as ${ }^{1}$

$$
\begin{aligned}
\frac{d \Gamma}{d E d \Omega} \sim & \left(1+A_{0} \frac{\langle\mathbf{I}\rangle}{I} \cdot \frac{\mathbf{p}}{E}\right) \\
& +\xi_{1}\left(1+\xi_{A}\left(\hat{\mathbf{p}} \cdot \frac{\langle\mathbf{I}\rangle}{I}\right)\right) \hat{\mathbf{p}} \hat{\mathbf{n}}+\xi_{2} \frac{\langle\mathbf{I}\rangle}{I} \hat{\mathbf{n}}+\xi_{3} \hat{p}_{i}\left(\frac{\langle\mathbf{I}\rangle}{I}\right)_{j} \rho^{i j}
\end{aligned}
$$

in which $\mathbf{p}$ and $\mathrm{E}$ are the momentum and energy of the decay electron, and the $\xi_{1,2,3, A}$ describe the coupling strengths to the hypothetical vector and rank 3 tensor fields $\hat{\mathbf{n}}$ and $\rho^{i j}$ which violate Lorentz invariance by defining a "preferred" reference frame. The first term in (1) describes the Standard Model contribution to the decay rate. Any non-zero $\xi$ would indicate a violation of Lorentz invariance. Precise measurements of the decay rate as a function of the experimental system's rotation against the preferred reference frame can therefore be used to probe a possible violation of Lorentz invariance by looking for changes in the decay rate when the experimental system rotates on the earth (daily variations), when the experimental system rotates with the earth around the sun (annual variations), or by deliberately reorienting the experimental system on shorter timescales (i.e. by periodical reorientation of the nuclear spin I).

\section{Testing Lorentz symmetry at KVI}

We are setting up a test of Lorentz invariance using short-lived alkali isotopes produced with the AGOR cyclotron at KVI. To reduce effects of contamination from other isotopes, it is desirable to stop the atoms in a buffer gas and transport them away from the production beamline. Nuclear polarization is obtained via optical pumping using a weak magnetic field and circularly polarized laser light. Changing the circular polarization of the laser light will reverse the nuclear polarization. Detecting a change in the decay lifetime when switching the nuclear polarization would indicate the presence of violation of Lorentz invariance. Figure 1 depicts a simulation of decay rate over time assuming a change in the decay lifetime at each reversal of the nuclear spin. A signal of this type can be looked for detecting the $\beta$-particle (or the corresponding annihilation photons at $511 \mathrm{keV}$ for $\beta^{+}$decays) or photons from decays of daughter particles. In addition, monitoring the fluorescence produced in the optical pumping process gives an indication of the number of atoms

$\overline{1}$ J. P. Noordmans, R. G. E. Timmermans (private communication) 
Fig. 1 Simulation of the decay rate of ${ }^{80} \mathrm{Rb}$ under periodic reversal of nuclear spin, assuming a change in decay lifetime of $\pm 50 \%$. The expected effect in lifetime change in the experiment is smaller by many magnitudes. Solid and dashed lines represent different sign $(+$ or -$)$ of the change in lifetime for a given orientation of the nuclear spin

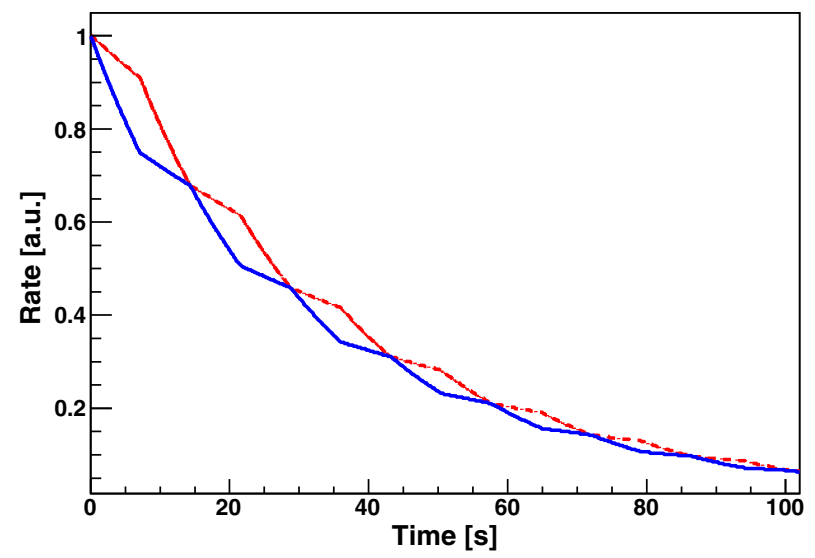

which have not decayed yet and may therefore also be used to reveal changes in the decay rate.

Efforts up to now are concentrated on the case of ${ }^{80} \mathrm{Rb}$ atoms $\left(\tau_{1 / 2}=33.4 \mathrm{~s}\right.$, see http://www.nndc.bnl.gov/chart/chartNuc.jsp). Other isotopes like ${ }^{21} \mathrm{Na}\left(\tau_{1 / 2}=22.5 \mathrm{~s}\right)$ or ${ }^{20} \mathrm{Na}\left(\tau_{1 / 2}=0.448 \mathrm{~s}\right)$ are also possible candidates to study Lorentz invariance in weak decays. One common requirement to all candidates is the pure Gamov-Teller decay. In this way, one can use the parity-violating asymmetry in the emission of the $\beta$-particle to monitor the nuclear polarization of the atoms.

\subsection{Production and transport}

${ }^{80} \mathrm{Rb}$ is produced in situ via a ${ }^{82} \mathrm{Kr}(\mathrm{p}, 3 \mathrm{n}){ }^{80} \mathrm{Rb}$ reaction using a 10 bar krypton gas target. In this configuration, one can obtain up to $10^{9}$ decays/s. The large pressure of the krypton gas reduces the diffusion of the ${ }^{80} \mathrm{Rb}$ atoms and thus prevents the adsorption at the inner wall of the production target tube. This makes it possible to transport the radioactive isotopes with the buffer gas out of the production target by means of a small circulation pump. During one circulation cycle, the gas flows past a stack of thin titanium foils, which are stacked at distances of few $\mathrm{mm}$. The ${ }^{80} \mathrm{Rb}$ atoms diffuse to the surface of the foil, and are extracted from the buffer gas by adsorption at room temperature [4]. After pumping away the krypton gas, an electrical current through the titanium foils will heat them up to about $900 \mathrm{~K}$, releasing the rubidium atoms to the optical cell in which they will subsequently be spin-polarized. The removal of the krypton gas is mandatory because the spin-polarization needs much lower buffer gas pressures (typically 10-100 mbar) than the 10 bar needed to produce the rubidium atoms.

\subsection{Polarization via optical pumping and signal detection}

The rubidium atoms are polarized via "optical pumping" (see e.g. [5]) using a weak magnetic field and circularly polarized laser light with a wavelength corresponding to the D1 or D2 transition of rubidium (795 or $780 \mathrm{~nm}$ ). As shown in Fig. 2, the atoms will be forced into a "stretched" ground state with both the polarizations of the nucleus and the electron cloud aligned in the same direction along the magnetic 


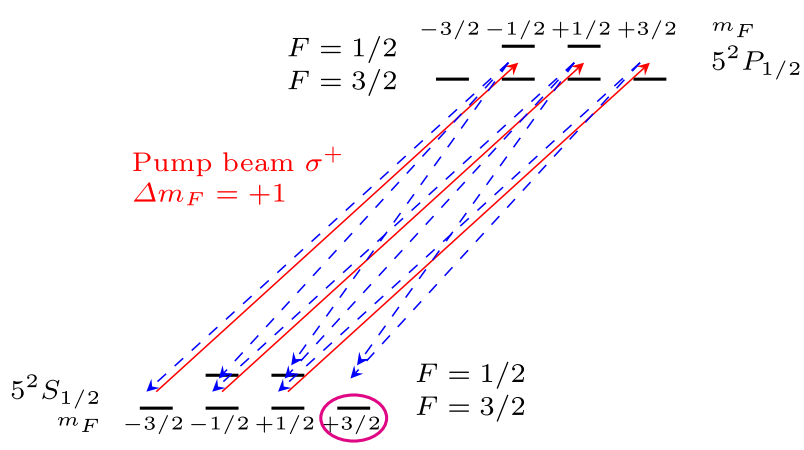

Fig. 2 Optical pumping of ${ }^{80} \mathrm{Rb}(\mathrm{I}=1)$ using the $5^{2} S_{1 / 2} \rightarrow 5^{2} P_{1 / 2}$ (D1) transition. After several pumping cycles, atoms will end up in the "stretched" ground state with $F=3 / 2, m_{F}=3 / 2$. Nuclear and electronic polarization are both aligned along the magnetic field lines. Switching the helicity from $\sigma^{+}$to $\sigma^{-}$will result in the atoms occupying the $F=3 / 2, m_{F}=-3 / 2$ ground state, with nuclear and electronic polarizations both flipped by $180^{\circ}$

Fig. 3 Experimental setup to test Lorentz invariance at KVI (courtesy C. J. G. Onderwater)

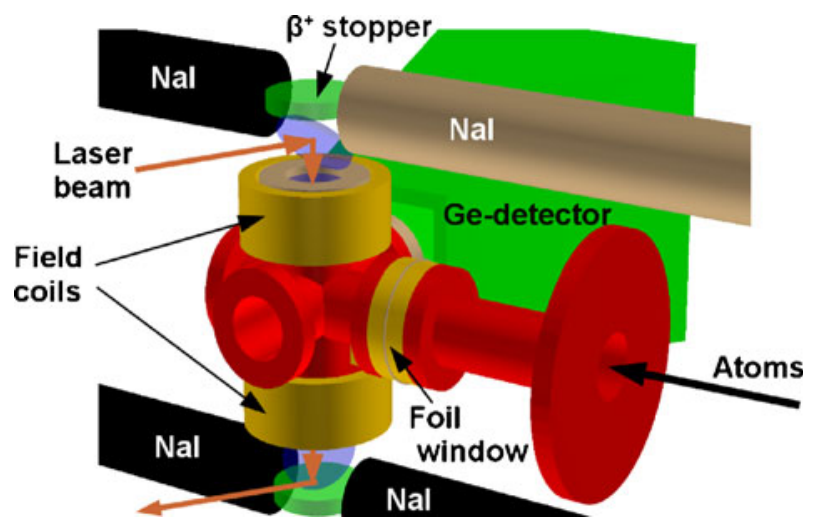

field lines. Changing the helicity of the laser light from $\sigma^{+}$to $\sigma^{-}$will flip the nuclear and electronic polarization by $180^{\circ}$. Helicity switching is obtained by splitting a laser beam evenly into a $\sigma^{+}$and a $\sigma^{-}$part and using a chopper wheel to rapidly switch between beams. Frequency stabilization of the laser is achieved by locking it to hyperfine transitions of stable rubidium in a vapor cell. The setup to detect the weak decay of the spin-polarized atoms is shown in Fig. 3. Two pairs of NaIdetectors (above and below the cell containing the radioactive atoms) are used to detect coincidences from $511 \mathrm{keV}$-photons produced by $\beta^{+}$particles emitted in the upward- or downward direction. The parity-violating asymmetry in the $\beta^{+}$-emission angle for spin-polarized atoms can be used to extract and monitor the amount of nuclear polarization created by the optical pumping process in the sample. A Gedetector is looking from the side at the cell and is used to observe the decay rate by detecting $\beta$-particles or photons from the decay of daughter particles (e.g. in the $25 \%$ of the cases when a ${ }^{80} \mathrm{Rb}$ atom decays to an excited state of ${ }^{80} \mathrm{Kr}$ ). The Ge-detector is thus providing the lifetime measurement used to test the Lorentz invariance. 


\section{Conclusions and outlook}

Lorentz invariance is a keystone of modern physics, and the observation of violation of Lorentz invariance would highly affect our current understanding of nature. To date, no compelling evidence for Lorentz invariance violation has been found. At KVI, we are currently setting up an experiment which will provide a unique test of Lorentz invariance using the weak decay of spin-polarized atoms. Violation of Lorentz invariance would manifest itself by a change in the decay rate of the atoms while reverting the orientation of their nuclear spin. The corresponding theoretical development for interpreting the observables within the framework of the Standard Model Extension is underway. Tests for the production and transport of ${ }^{80} \mathrm{Rb}$ have been carried out. A laser setup to create nuclear polarization via optical pumping has been built, and the design of the detection system is ready. The next steps are to construct the detection system and test it together with the optical setup using a clean beam of ${ }^{20} \mathrm{Na}$ from the TRI $\mu \mathrm{P}$ isotope separator.

Acknowledgements This research was supported by the Stichting voor Fundamenteel Onderzoek der Materie (FOM) under Program 114 (TRI $\mu$ P) and FOM projectruimte 08PR2636-1. The presented work has been done in collaboration with E. A. Dijck, J. P. Noordmans, C. J. G. Onderwater, R. G. E. Timmermans and H. W. Wilschut (all KVI Groningen).

Open Access This article is distributed under the terms of the Creative Commons Attribution License which permits any use, distribution, and reproduction in any medium, provided the original author(s) and source are credited.

\section{References}

1. Greenberg, O.W.: Phys. Rev. Lett. 89, 231602 (2002)

2. Colladay, D., Kostelecky, V.A.: Phys. Rev. D 58, 116002 (1998)

3. Kostelecky, V.A., Russell, N.: Rev. Mod. Phys. 83, 11 (2011)

4. Roßbach, H., Eichler, B.: Adsorption von Metallen auf metallische Oberflächen und Möglichkeiten ihrer Nutzung in der Kernchemie. ZfK Report Rossendorf (1984)

5. Demtröder, W.: Laser spectroscopy: Basic Concepts and Instrumentation. Springer, Berlin (1998) 